\title{
Plasma concentrations of vascular endothelial growth factor are associated with peripheral oedema in patients treated with thiazolidinedione
}

\author{
M. Emoto $\cdot$ N. Fukuda $\cdot$ Y. Nakamori $\cdot$ A. Taguchi • \\ S. Okuya $\cdot$ Y. Oka $\cdot$ Y. Tanizawa
}

Received: 20 April 2006 /Accepted: 24 April 2006/ Published online: 1 July 2006

(C) Springer-Verlag 2006

To the Editor Thiazolidinediones are widely used in patients with type 2 diabetes. In addition to lowering plasma glucose, this class of drugs may reduce the risk of cardiovascular diseases in patients with diabetes. Despite the beneficial effect of thiazolidinediones, their use is sometimes associated with peripheral oedema. This is the most frequent adverse effect leading to discontinued treatment with thiazolidinediones in Japan. However, the mechanism by which thiazolidinediones induce oedema remains to be established.

Recently, Guan et al. reported that, in a mouse model, thiazolidinediones caused fluid retention through peroxisome proliferator-activated receptor- $\gamma$ stimulation of renal salt absorption mediated by epithelial $\mathrm{Na}^{+}$channel $(\mathrm{ENaC})$ [1]. The authors speculated that an increase in plasma volume causes thiazolidinedione-induced peripheral oedema. We, however, doubt that this is the sole cause of oedema in humans. $\mathrm{ENaC}$ is a sodium channel located in collecting tubules. This channel is also the site of action of the hormone aldosterone, a mineralocorticoid that increases sodium reabsorption. Primary aldosteronism is a disease caused by hypersecretion of this hormone. Due to excess sodium reabsorption, the circulating blood volume increases. The

\footnotetext{
M. Emoto $\cdot$ N. Fukuda $\cdot$ Y. Nakamori $\cdot$ A. Taguchi $\cdot$ S. Okuya

Y. Tanizawa $(\bowtie)$

Division of Endocrinology, Metabolism, Hematological Sciences

and Therapeutics, Department of Bio-Signal Analysis,

Yamaguchi University Graduate School of Medicine,

1-1-1, Minami-Kogushi,

Ube 755-8505, Japan

e-mail: tanizawa@yamaguchi-u.ac.jp

Y. Oka

Division of Molecular Metabolism and Diabetes,

Tohoku University Graduate School of Medicine,

Sendai, Japan
}

resulting headache and hypertension are well documented. However, peripheral oedema does not usually develop [2]. This is a well-known outcome of this disease. Oedema, moreover, also does not develop as a result of the increased plasma volume in the syndrome of inappropriate secretion of antidiuretic hormone [3]. Antidiuretic horomone is a pituitary hormone that activates the water channel aquaporin 2 in collecting tubules, thereby increasing water reabsorption. These observations in relation to endocrine diseases suggest that blood volume expansion is not sufficient for peripheral oedema to develop.

We would like to highlight another important factor that might also be involved in the process. Previously, we reported that thiazolidinediones increase the plasma levels of vascular endothelial growth factor (VEGF) [4]. Since VEGF is a potent vascular permeability factor, an increase in VEGF levels may contribute to thiazolidinedioneinduced oedema. However, because of the difficulty of quantifying the severity of oedema, no one has demonstrated a clear correlation between peripheral oedema and plasma VEGF levels. Assessing the presence of peripheral oedema by body weight can be misleading, because thiazolidinediones increase body fat mass [5] and circulating blood volume [1]. In our previous report, for example, a weak correlation between body weight and plasma VEGF concentration was observed only in female patients, but not in male patients or groups comprising both sexes [4].

Here, we demonstrate a strong correlation between clinically apparent peripheral oedema and plasma VEGF concentration. Oedema is defined as a clinically apparent increase in the interstitial fluid volume. Thus, we judged the existence of peripheral oedema by the presence of pitting after pressure was applied to the bilateral lower extremities. We re-evaluated the clinical data collected for our previous study [4]. Briefly, a total of 30 patients with type 2 diabetes 


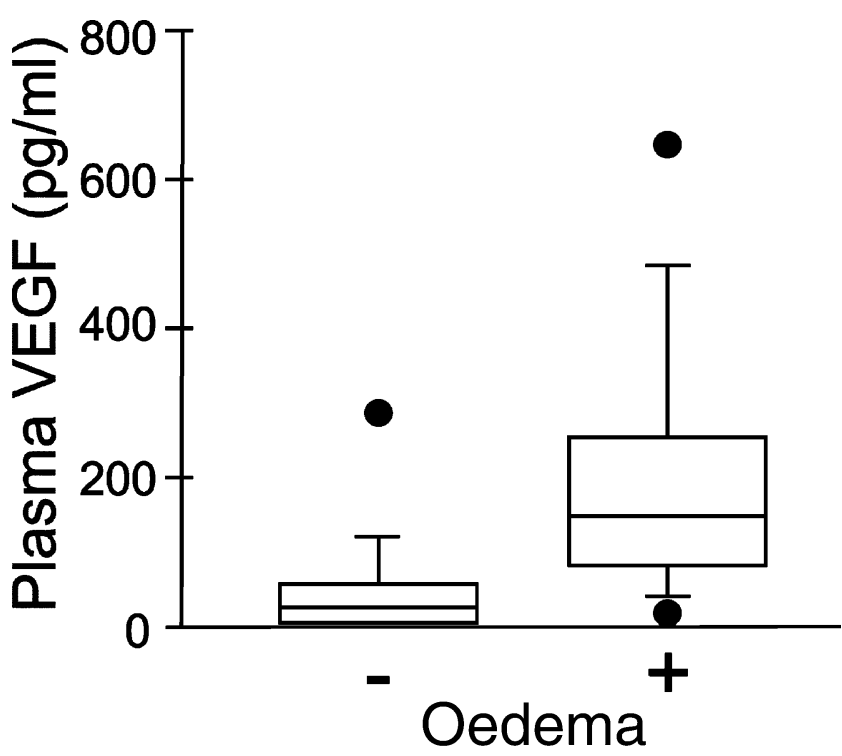

Fig. 1 Plasma vascular endothelial growth factor $(V E G F)$ increase in patients with peripheral oedema. Plasma VEGF concentrations were measured in patients with and without peripheral oedema, as determined by physical examination. Concentrations were elevated in patients with $(+)$ peripheral oedema. Data are medians, 70th and 90th percentiles. $p=0.0011, n=12$ in each group, Mann-Whitney test

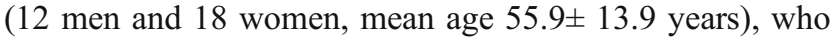
had already been treated with troglitazone (the first thiazolidinedione compound) $400 \mathrm{mg}$ daily for 3-6 months, participated in the study. No one had a history of congestive heart failure or was receiving combination therapy with insulin. Patients with cancer, liver cirrhosis, nephrotic syndrome or varicose veins were excluded. We measured total circulating $\mathrm{VEGF}_{165 / 121}$ isoforms in platelet-poor EDTA-plasma. Based on clinical records, the patients treated with troglitazone were divided into two groups in a binary manner. One comprised patients who had peripheral oedema determined by physical examination; the other patients who did not. In some patients, we were uncertain whether they had oedema or not. These patients were excluded from the study (plasma VEGF $66.1 \mathrm{pg} / \mathrm{ml}$ [median]; $n=6$ ). As shown in Fig. 1, the plasma VEGF 165/121 concentration was significantly higher in patients with than in those without oedema (with $164.5 \mathrm{pg} / \mathrm{ml}$, without $33.2 \mathrm{pg} / \mathrm{ml}$ [median], respectively; $p=0.0011 ; n=12$ in each group). None of the patients had peripheral oedema at the outset, either in the troglitazone group, or in the control groups (patients treated by diet alone, sulfonylurea, and insulin; $n=10$ in each group). Moreover, reversal of oedema and plasma VEGF concentration was observed after discontinuation of treatement with troglitazone. Our present data suggest that increased plasma VEGF is one of the major factors of thiazolidinedione-induced peripheral oedema.
On the other hand, it is also true that the patients treated with thiazolidinediones had mild haemodilution [6]. This clinical observation suggests that other fluid dynamic factors may be involved in the development of oedema. VEGF disrupts vascular barrier function, and endothelial cells exposed to VEGF allow passage of particles of different sizes by a variety of mechanisms. In this pathological condition, vascular leakage is thought to be dependent on relative pressure gradients between the vascular and extra-vascular compartments [7]. If renal salt absorption is increased and microvascular pressure is high relative to interstitial pressure, capillary leakage may be clinically evident. These pathophysiological consequences are consistent with the idea that both VEGF-enhanced vascular permeability and $\mathrm{ENaC}$-mediated fluid retention are necessary for thiazolidinediones to induce peripheral oedema. Other thiazolidinediones such as pioglitazone also increase plasma VEGF concentrations [8, unpublished observation by the authors]. However, Tooke et al. argued against this point in a recent online publication [9]. In any case, since oedema is one of the major factors for discontinuing treatment with thiazolidinediones, further studies are needed to develop safer and better tolerated new thiazolidinediones.

In summary, we hypothesised that the mechanism of thiazolidinedione-induced peripheral oedema involves the synergistic effect of water retention and hyperpermeability.

\section{References}

1. Guan Y, Hao C, Cha DR et al (2005) Thiazolidinediones expand body fluid volume through PPAR $\gamma$ stimulation of ENaC-mediated renal salt absorption. Nat Med 11:861-866

2. Williams GH, Dluhy RG (2005) Disorders of the adrenal cortex. In: Kasper DL et al. (eds) Harrison's principles of internal medicine, 16th edn. McGraw-Hill, New York, pp 2138-2140

3. Bartter FC, Schwartz WB (1967) The syndrome of inappropriate secretion of antidiuretic hormone. Am J Med 42:790-806

4. Emoto M, Anno T, Sato $Y$ et al (2001) Troglitazone treatment increases plasma vascular endothelial growth factor in diabetic patients and its mRNA in 3T3-L1 adipocytes. Diabetes 50:11661170

5. Kelly IE, Han TS, Walsh K, Lean ME (1999) Effects of a thiazolidinedione compound on body fat and fat distribution of patients with type 2 diabetes. Diabetes Care 22:288-293

6. Hollenberg NK (2003) Considerations for management of fluid dynamic issues associated with thiazolidinediones. Am J Med 115 (Suppl 8A):111S-115S

7. Weis SM, Cheresh DA (2005) Pathophysiological consequences of VEGF-induced vascular permeability. Nature 437:497-504

8. Baba T, Shimada K, Neugebauer S et al (2001) The oral insulin sensitizer, thiazolidinedione, increases plasma vascular endothelial growth factor in type 2 diabetic patients. Diabetes Care 24:953-954

9. Tooke JE, Elston LM, Gooding KM et al (2006) The insulin sensitiser pioglitazone does not influence skin microcirculatory function in patients with type 2 diabetes treated with insulin. Diabetologia 49:1064-1070 